\title{
Photoionization of the alkali molecular ions in geo-cosmical plasmas
}

\author{
Lj.M. Ignjatović ${ }^{1}$, V.A. Srećković ${ }^{1}$ and M.S. Dimitrijević ${ }^{2,3}$ \\ 1 Institute of Physics Belgrade, BU, Pregrevica 118, 11080 Belgrade, Serbia \\ (E-mail: vlada@ipb.ac.rs) \\ 2 Astronomical Observatory, Volgina 7, 11060 Belgrade, Serbia (E-mail: \\ mdimitrijevic@aob.rs) \\ 3 Sorbonne Université, Observatoire de Paris, Université PSL, CNRS, \\ LERMA, F-92190, Meudon, France
}

Received: July 16, 2019; Accepted: September 29, 2019

\begin{abstract}
Opacities of the solar and stellar atmospheres are caused by a large number of radiative processes. Within development of more sophisticated stellar atmosphere models, we can further investigate known processes and include all processes not considered before. We calculate the average cross-section and rate coefficients for the photodissociation of the alkali molecular ions $\mathrm{Li}_{2}^{+}, \mathrm{Na}_{2}^{+}$ and $\mathrm{LiNa}^{+}$ready for further use with a particular accent to the applications for astro plasma research and low temperature laboratory plasma research.
\end{abstract}

Key words: Atomic processes - Line: profiles - Plasmas - ISM: clouds

\section{Introduction}

The influence of some hydrogen and helium radiative processes on the optical characteristics of the stellar atmospheres was already established (see e.g. Fontenla et al., 2006; Ignjatović et al., 2009). Here we keep in mind such symmetric and non-symmetric radiative processes as photoabsorption/emission described in Srećković et al. (2017) and Marinković et al. (2017)

Recently, in the papers Mihajlov et al. (2007); Ignjatović et al. (2014); Srećković et al. (2014); Babb (2015); Heays et al. (2017) has been pointed out that the photodissociation of the diatomic molecular ion in the symmetric and nonsymmetric cases, are of astrophysical relevance and could be important in modeling of specific stellar atmosphere layers and they should be included in some chemical models. In the symmetric case, it was considered the processes of molecular ion photodissociation and inverse photoassociation:

$$
h \nu+A_{2}^{+} \Longleftrightarrow A+A^{+},
$$

where $A$ and $A^{+}$are atom and ion in their ground states, and $A_{2}^{+}$is molecular-ion in the ground electronic state. In the non-symmetric case, the similar processes of photodissociation/ photoassociation are:

$$
h \nu+A B^{+} \Longleftrightarrow A^{+}+B,
$$


where $B$ is an atom whose ionization potential is less than the corresponding value for atom $A$. $A B^{+}$is also molecular-ion in the ground electronic state.

For the solar atmosphere, $A$ designates atom $\mathrm{H}(1 \mathrm{~s})$ and $B$ one of the relevant metal atoms $\mathrm{Si}, \mathrm{Ca}, \mathrm{Na}, \mathrm{Mg}$. Also there are cases where $A=\mathrm{He}$, and $B=\mathrm{H}$, Mg, Si, Na, Ca (Mihajlov et al., 2007; Ignjatović et al., 2014; Srećković et al., 2014). For the helium-rich white dwarf atmospheres $A$ designate $\mathrm{He}\left(1 \mathrm{~s}^{2}\right)$ and $B$ $\mathrm{H}(1 \mathrm{~s})$, and eventually oxygen or carbon (Ignjatović et al., 2009; Mihajlov et al., 2013). Recently, Srećković et al. $(2017,2018)$ show the importance of including the symmetric processes (1) with $A=\mathrm{H}(1 \mathrm{~s})$ in the solar atmosphere models like Fontenla et al. (2006). Also, for modeling the DB white dwarf atmospheres results for case $A=\mathrm{He}\left(1 s^{2}\right)$ have been used (Koester, 2010).

It is well known (Puy et al., 2007) that the chemical composition of the primordial gas consists of electrons and species such as: helium - $\mathrm{He}, \mathrm{He}^{+}, \mathrm{He}^{2+}$ and $\mathrm{HeH}^{+}$; hydrogen - $\mathrm{H}, \mathrm{H}^{-}, \mathrm{H}^{+}, \mathrm{H}_{2}^{+}$and $\mathrm{H}_{2}$; deuterium - $D, D^{+}, \mathrm{H} D, \mathrm{H} D^{+}$ and $\mathrm{H} D^{-}$; lithium $-\mathrm{Li}, \mathrm{Li}^{+}, \mathrm{Li}^{-}, \mathrm{LiH}^{-}$and $\mathrm{LiH}^{+}$. One can see that among them are species like molecular ions $A_{2}^{+}$and $A B^{+}$whose role in the primordial star formation is important (see also Coppola et al., 2011; Glover et al., 2014).

The average cross-section for the photodissociation and the corresponding spectral absorption coefficients of the alkali molecular ions $\mathrm{Li}_{2}^{+}, \mathrm{Na}_{2}^{+}$and $\mathrm{LiNa}^{+}$ are calculated for the wide region of temperatures and wavelengths. The presented results are ready for further use with a particular accent to the applications for astro plasma research and low temperature laboratory plasma research created in gas discharges, where plasma conditions may be favorable for processes investigated here (Pichler et al., 2017; Beuc et al., 2018).

We describe in Section 2 the methods of calculation and then in Section 3 the results of the calculation. The conclusions are presented in Section 4.

\section{The methods of calculation}

In accordance with the Ignjatović et al. (2014) the mean thermal photodissociation cross section, is

$$
\sigma^{(p h d)}(\lambda, T)=\frac{\sum_{J, v}(2 J+1) e^{\frac{-E_{J, v}}{k T}} \cdot \sigma_{J, v}(\lambda)}{\sum_{J, v}(2 J+1) e^{\frac{-E_{J, v}}{k T}}},
$$

and $\sigma_{J, v}(\lambda)$ is the partial photodissociation cross-section for the ro-vibrational states with given quantum numbers $J$ and $v$, and $E_{J, v}$ - the energies of these states with the respect to the ground ro-vibrational states. Here $E_{J, v}=E_{d i s}+$ $\epsilon_{J, v}$, where $E_{d i s}$ is the dissociative energy of the molecular ion, and the energies $\epsilon_{J, v}<0$ are determined as in Srećković et al. (2014); Srećković et al. (2017) together with the wave functions of the considered ro-vibrational states. Within 


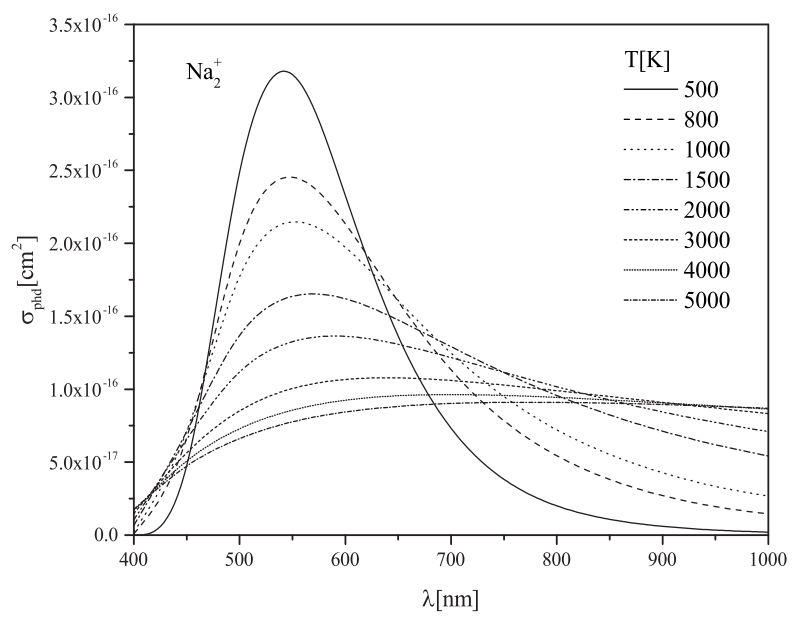

Figure 1. The behaviour of the averaged cross-section $\sigma_{p h d}(\lambda, T)$ for photodissociation of the $\mathrm{Na}_{2}^{+}$molecular ion, as a function of $\lambda$ and $T$.

the dipole approximation the partial cross-sections $\sigma_{J, v}(\lambda)$ are given by

$$
\begin{aligned}
& \sigma_{J, v}(\lambda)=\frac{8 \pi^{3}}{3 \lambda}\left[\frac{J+1}{2 J+1}\left|D_{J, v ; J+1, E_{i m p}^{\prime}}\right|^{2}\right. \\
& \left.+\frac{J}{2 J+1}\left|D_{J, v ; J-1, E_{i m p}^{\prime}}\right|^{2}\right], \\
& D_{J, v ; J \pm 1, E_{\text {imp }}^{\prime}}=<i n, J, v ; R\left|D_{i n, f i n}(R)\right| f i n, J \pm 1, E^{\prime}>,
\end{aligned}
$$

where $E^{\prime}=\epsilon_{J, v}+\varepsilon_{\lambda}, E_{i m p}^{\prime}$ and $E^{\prime}$ and $D_{i n, f i n}(R)$ are given in Srećković et al. (2014). $D_{i n ; f i n}(R)$ is the electronic dipole matrix element

$$
D_{i n ; f i n}(R)=<i n ; R|\mathbf{D}(R)| \text { fin } ; R>,
$$

where $\mathbf{D}$ is the operator of the dipole moment of the considered system.

The photodissociation spectral rate coefficient can be calculated using expression

$$
K(\lambda, T)=\sigma^{(p h d)}(\lambda, T) \cdot \chi^{-1}(T),
$$

where the factor $\chi(T)=N(A) N\left(B^{+}\right) / N\left(A B^{+}\right)$is given by the relation

$$
\chi(T)=\frac{g_{1} g_{2}}{g_{12}}\left(\frac{\mu k T}{2 \pi \hbar^{2}}\right)^{\frac{3}{2}} \cdot \frac{1}{\sum_{J, v}(2 J+1) e^{\frac{E_{d i s}-E_{J, v}}{k T}}},
$$

where $g_{1}, g_{2}$ and $g_{12}$ are the electronic statistical weights of the species, $A, B^{+}$ and $A B^{+}$respectively, and $\sigma^{(p h d)}(\lambda, T)$ is given by Eqs. (3)-(5). 


\section{Results and discussion}

The results for the average photodissociation cross-section $\sigma^{(p h d)}(\lambda, T)$ on the example of the $\mathrm{Na}_{2}^{+}$molecular ion are illustrated by Fig. 1 . The curves in this figure show the behavior of $\sigma^{(p h d)}(\lambda, T)$ as a function of $\lambda$ for a wide range of temperatures $T$, which are relevant for modeling geo-cosmical plasmas (Klyucharev et al., 2007). The values of the $\mathrm{Li}_{2}^{+}, \mathrm{Na}_{2}^{+}, \mathrm{LiNa}^{+}$rate coefficient $K(\lambda, T)$, defined by Eq. (7), are presented in Fig. 2 for $500 \mathrm{~K} \leq T \leq 5000 \mathrm{~K}$ in order to enable easier use (interpolation) of this results (see e.g. Ignjatović et al., 2019). This allows direct calculation of the spectral absorption coefficients during the process of applying an atmosphere model with the given parameters and composition of plasma.

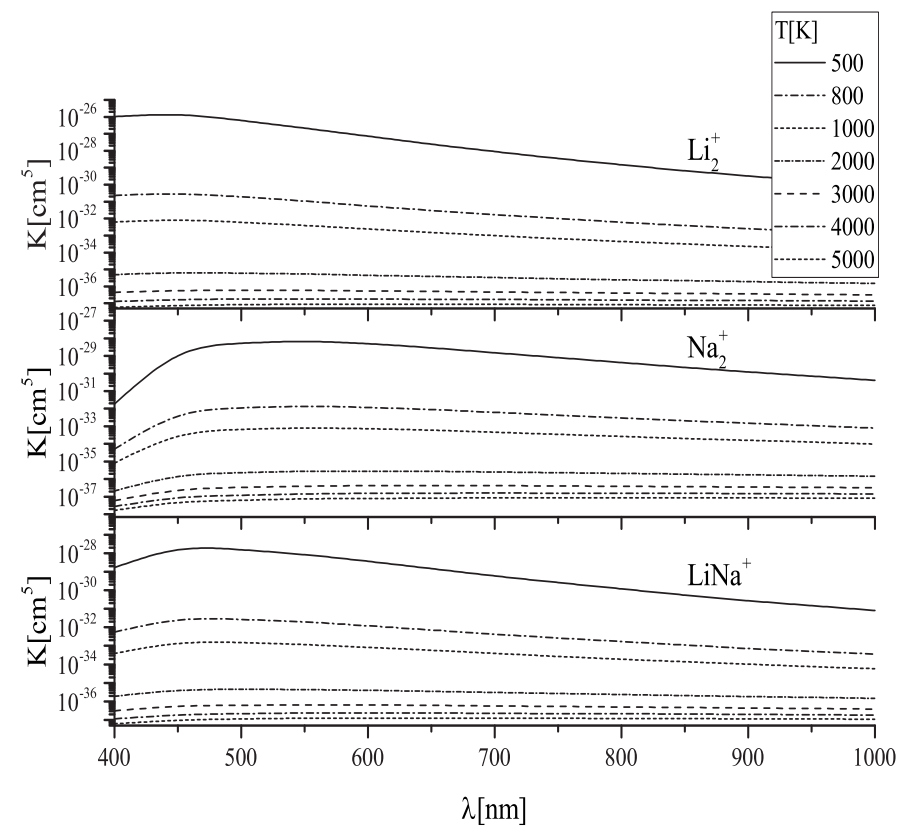

Figure 2. The plot of the spectral rate coefficients for photodissociation of the $\mathrm{Li}_{2}^{+}$, $\mathrm{Na}_{2}^{+}, \mathrm{LiNa}^{+}$, as a function of $\lambda$ and $T$.

These figures show that exist a noticeable difference between temperature dependence of the mean thermal photo-ionization cross section and the corresponding spectral rate coefficient. The results obtained during this investigation will be implemented in the MolD database (Vujčić et al., 2015; Marinković et al., 2017, 2019). 


\section{Conclusions and Discussion}

We determine the average cross-section for the photodissociation processes and the corresponding spectral rate coefficient of the alkali molecular ions $\mathrm{Li}_{2}^{+}, \mathrm{Na}_{2}^{+}$, $\mathrm{LiNa}^{+}$in the region $\lambda \leq 1000 \mathrm{~nm}$. The calculated data are useful for modelling and investigation of cool stars, especially brown and white dwarfs, lithium stars, Io's cloud of sodium atoms, and primordial gas containing Li atoms and ions. Also, the presented results are also important in theoretical and laboratory spectroscopic plasmas research.

Acknowledgements. This work is made within projects of the MESTD of Serbia, grant numbers 176002, III44002.

\section{References}

Babb, J. F., State resolved data for radiative association of $\mathrm{H}$ and $\mathrm{H}^{+}$and for Photodissociation of $\mathrm{H}_{2}^{+}$. 2015, Astrophys. J. Suppl. Ser., 216, 21

Beuc, R., Peach, G., Movre, M., \& Horvati, B., Lithium, sodium and potassium resonance lines pressure broadened by helium atoms. 2018, Astron. Astrophys. Trans., 30, 315

Coppola, C. M., Longo, S., Capitelli, M., Palla, F., \& Galli, D., Vibrational level population of $\mathrm{H}_{2}$ and $\mathrm{H}_{2}{ }^{+}$in the early universe. 2011, Astrophys. J. Suppl. Ser., 193,7

Fontenla, J. M., Avrett, E., Thuillier, G., \& Harder, J., Semiempirical Models of the Solar Atmosphere. I. The Quiet- and Active Sun Photosphere at Moderate Resolution. 2006, Astrophys. J., 639, 441

Glover, S. C. O., Chluba, J., Furlanetto, S. R., Pritchard, J. R., \& Savin, D. W., Chapter Three - Atomic, Molecular, and Optical Physics in the Early Universe: From Recombination to Reionization. 2014, Advances in Atomic Molecular and Optical Physics, 63, 135

Heays, A., Bosman, A., \& van Dishoeck, E., Photodissociation and photoionisation of atoms and molecules of astrophysical interest. 2017, Astron. Astrophys., 602, A105

Ignjatović, L. M., Mihajlov, A. A., Sakan, N. M., Dimitrijević, M. S., \& Metropoulos, A., The total and relative contribution of the relevant absorption processes to the opacity of DB white dwarf atmospheres in the UV and VUV regions. 2009, Mon. Notices Royal Astron. Soc., 396, 2201

Ignjatović, L. M., Mihajlov, A. A., Srećković, V. A., \& Dimitrijević, M. S., Absorption non-symmetric ion-atom processes in helium-rich white dwarf atmospheres. 2014, Mon. Notices Royal Astron. Soc., 439, 2342

Ignjatović, L. M., Srećković, V., \& Dimitrijević, M., The collisional atomic processes of Rydberg alkali atoms in geo-cosmical plasmas. 2019, Mon. Notices Royal Astron. Soc., 483, 4202 
Klyucharev, A. N., Bezuglov, N. N., Matveev, A. A., et al., Rate coefficients for the chemi-ionization processes in sodium- and other alkali-metal geocosmical plasmas. 2007, New.Astr.Rev., 51, 547

Koester, D., White dwarf spectra and atmosphere models . 2010, Mem. S.A.It., 81, 921

Marinković, B. P., Jevremović, D., Srećković, V. A., et al., BEAMDB and MolDdatabases for atomic and molecular collisional and radiative processes: Belgrade nodes of VAMDC. 2017, Eur. Phys. J. D, 71, 158

Marinković, B. P., Srećković, V. A., Vujčić, V., et al., BEAMDB and MOLDDatabases at the Serbian Virtual Observatory for Collisional and Radiative Processes. 2019, Atoms, 7, 11

Mihajlov, A., Ignjatović, L. M., Sakan, N., \& Dimitrijević, M., The influence of $\mathrm{H}_{2}^{+}$photo-dissociation and $\left(\mathrm{H}+\mathrm{H}^{+}\right)$-radiative collisions on the solar atmosphere opacity in UV and VUV regions. 2007, Astron. Astrophys., 469, 749

Mihajlov, A. A., Ignjatović, L. M., Srećković, V. A., Dimitrijević, M. S., \& Metropoulos, A., The non-symmetric ion-atom radiative processes in the stellar atmospheres. 2013, Mon. Notices Royal Astron. Soc., 431, 589

Pichler, G., Makdisi, Y., Kokaj, J., et al., Superheating effects in line broadening of dense alkali vapors. 2017, in J. Phys. Conf. Ser., Vol. 810, J. Phys. Conf. Ser., 012013

Puy, D., Dubrovich, V., Lipovka, A., Talbi, D., \& Vonlanthen, P., Molecular fluorine chemistry in the early Universe. 2007, Astron. Astrophys., 476, 685

Srećković, V., Dimitrijević, M., Ignjatović, L., Bezuglov, N., \& Klyucharev, A., The Collisional Atomic Processes of Rydberg Hydrogen and Helium Atoms: Astrophysical Relevance. 2018, Galaxies, 6, 72

Srećković, V. A., Ignjatović, L. M., Jevremović, D., Vujčić, V., \& Dimitrijević, M. S., Radiative and Collisional Molecular Data and Virtual Laboratory Astrophysics. 2017, Atoms, 5, 31

Srećković, V. A., Mihajlov, A. A., Ignjatović, L. M., \& Dimitrijević, M. S., Ion-atom radiative processes in the solar atmosphere: Quiet Sun and sunspots. 2014, Adv. Space Res., 54, 1264

Vujčić, V., Jevremović, D., Mihajlov, A. A., et al., MOL-D: A Collisional Database and Web Service within the Virtual Atomic and Molecular Data Center. 2015, J. Astrophys. Astron., 36, 693 